\title{
Structure-Based Approaches to Classify the Functional Impact of ZBTB18 Missense Variants in Health and Disease
}

\section{SUPPORTING INFORMATION}

Steven Blake, ${ }^{1,2,3}$ Isabel Hemming, ${ }^{1,2,4}$ Julian Heng, ${ }^{1,2}$ Mark Agostino*1,3,5

${ }^{1}$ Curtin Health Innovation Research Institute, Curtin University, Bentley, Western Australia, Australia

${ }^{2}$ Ralph and Patricia Sarich Neuroscience Research Institute, Nedlands, Western Australia, Australia ${ }^{3}$ School of Pharmacy and Biomedical Sciences, Curtin University, Bentley, Western Australia,

Australia

${ }^{4}$ The Faculty of Health and Medical Sciences, Medical School, The University of Western Australia, Crawley, Western Australia, Australia

${ }^{5}$ Curtin Institute for Computation, Curtin University, Bentley, Western Australia, Australia

*To whom correspondence should be addressed: Mark.Agostino@curtin.edu.au 

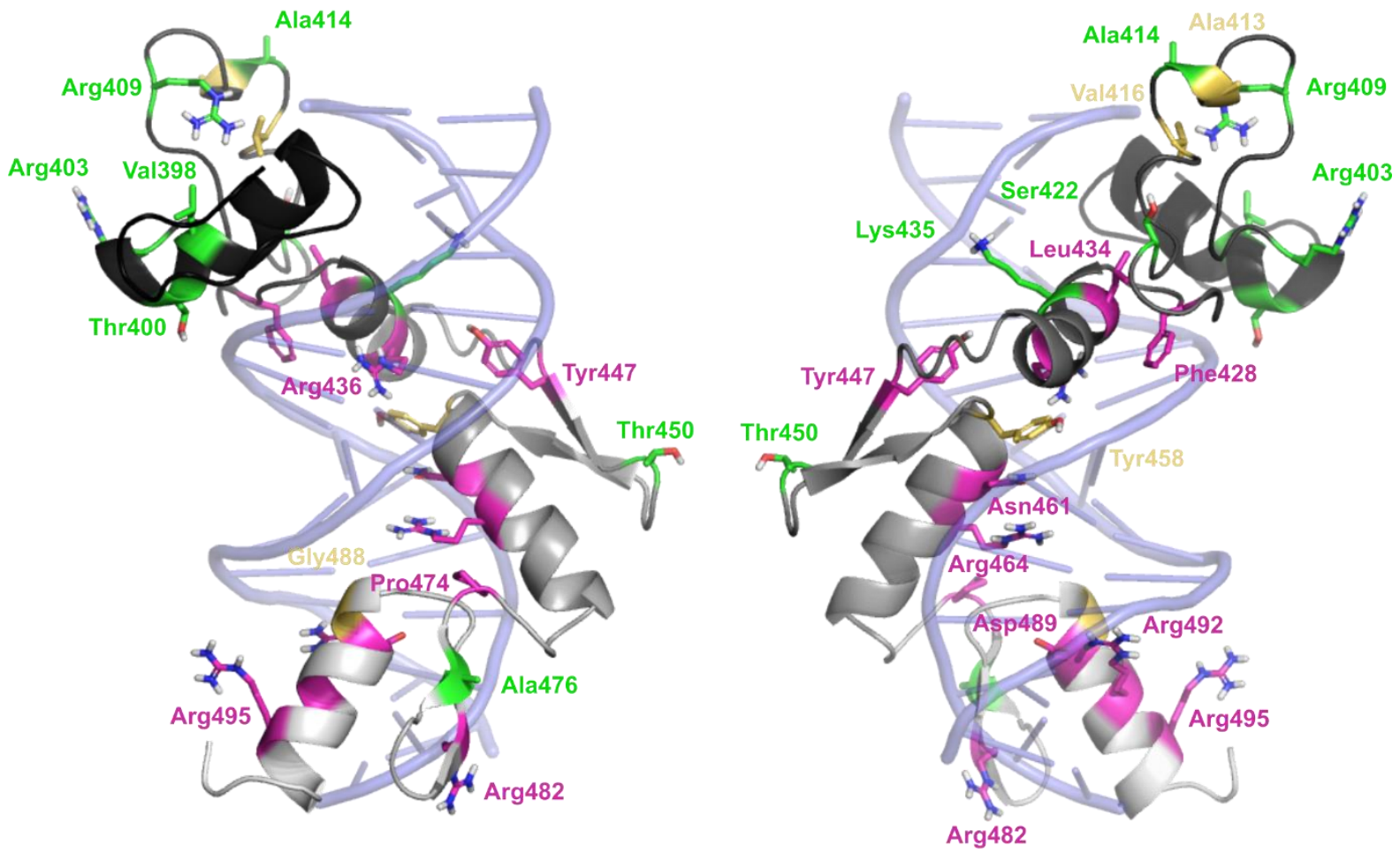

Figure S1. Locations of ZBTB18 variants examined in this study, illustrated on the energyminimised wildtype ZBTB18 complex with Rnd2-E1. Zinc fingers are shown from top to bottom in sequential order, with the first and fourth zinc fingers facing the viewer in the left panel, and the second and third zinc fingers facing the viewer in the right panel (rotated 180 degrees in the y-axis relative to the left panel). Sites at which disease-associated variants are found are shown in magenta, sites at which general population variants are found are shown in green, and sites at which additional variants for testing are found are shown in yellow. 
Table S2. Rnd2 and Id2 DNA enhancer motifs used in this study. Residues indicated in bold deviate from the canonical DNA sequence to which ZBTB18 optimally binds.

\begin{tabular}{|l|l|}
\hline Rnd2-E1 & 5'-TAACAGATGTCTGTC-3' \\
3'-ATTGTCTACAGACAG-5' \\
\hline \multirow{2}{*}{ Rnd2-E2 } & 5'-GGGCAGATGGGAGTA-3' \\
\hline & 3'-CCCGTCTACCCTCAT-5' \\
\hline Id2-Bs1 1 & 5'-GCACATCTGGCGCAA-3' \\
\hline & 3'-CGTGTAGACCGCGTT-5' \\
\hline Id2-Bs2 & 3'-AGACAGCTGTGTCCA-3' \\
\hline
\end{tabular}


Table S3. Energetic data determined by thermodynamic integration for ZBTB18 variants bound to Rnd2-E1. ${ }^{\text {a }}$

\begin{tabular}{|c|c|c|c|c|c|c|c|}
\hline \multirow[b]{2}{*}{$\begin{array}{l}\text { ZBTB18 } \\
\text { Variant }\end{array}$} & \multicolumn{3}{|c|}{ Bound } & \multicolumn{3}{|c|}{ Unbound } & \multirow[b]{2}{*}{$\begin{array}{c}\Delta \Delta \mathrm{G} \\
(\mathrm{kcal} / \mathrm{mol})\end{array}$} \\
\hline & $\begin{array}{l}\Delta \text { G Forward } \\
\text { (kcal/mol) }\end{array}$ & $\begin{array}{l}\Delta \text { G Reverse } \\
\text { (kcal/mol) }\end{array}$ & $\begin{array}{l}\Delta G \text { Average } \\
\text { (kcal/mol) }\end{array}$ & $\begin{array}{c}\Delta G \text { Forward } \\
\text { (kcal/mol) }\end{array}$ & $\begin{array}{l}\Delta \text { G Reverse } \\
\text { (kcal/mol) }\end{array}$ & $\begin{array}{c}\Delta G \text { Average } \\
\text { (kcal/mol) }\end{array}$ & \\
\hline L398V & $+1.48 \pm 0.21$ & $+2.77 \pm 0.12$ & $+2.13 \pm 0.24$ & $+1.09 \pm 0.39$ & $+3.04 \pm 0.33$ & $+2.07 \pm 0.51$ & $+0.06 \pm 0.57$ \\
\hline $\mathrm{T} 400 \mathrm{Mb}$ & $+32.66 \pm 0.44$ & $+30.43 \pm 0.39$ & $+31.55 \pm 0.59$ & $+30.32 \pm 0.20$ & $+31.65 \pm 0.79$ & $+30.99 \pm 0.81$ & $+0.56 \pm 1.00$ \\
\hline R403C & $+175.64 \pm 0.22$ & $+176.15 \pm 0.18$ & $+175.90 \pm 0.28$ & $+174.91 \pm 0.11$ & $+176.74 \pm 0.26$ & $+175.83 \pm 0.28$ & $+0.07 \pm 0.40$ \\
\hline R409C & $+173.42 \pm 0.20$ & $+174.01 \pm 0.28$ & $+173.72 \pm 0.34$ & $+172.41 \pm 0.35$ & $+174.54 \pm 0.24$ & $+173.48 \pm 0.42$ & $+0.24 \pm 0.55$ \\
\hline A414T & $-29.28 \pm 0.30$ & $-27.67 \pm 0.16$ & $-28.48 \pm 0.34$ & $-29.58 \pm 0.06$ & $-27.27 \pm 0.19$ & $-28.43 \pm 0.20$ & $-0.05 \pm 0.39$ \\
\hline S422L & $-7.22 \pm 0.13$ & $-7.38 \pm 0.10$ & $-7.30 \pm 0.16$ & $-7.12 \pm 0.11$ & $-7.69 \pm 0.11$ & $-7.41 \pm 0.16$ & $+0.11 \pm 0.23$ \\
\hline F428C & $+1.57 \pm 0.39$ & $+3.75 \pm 0.23$ & $+2.66 \pm 0.45$ & $-1.84 \pm 0.15$ & $+0.97 \pm 0.19$ & $-0.44 \pm 0.24$ & $+3.10 \pm 0.51$ \\
\hline K435R & $-158.71 \pm 0.17$ & $-157.84 \pm 0.24$ & $-158.28 \pm 0.29$ & $-159.78 \pm 0.50$ & $-157.93 \pm 0.10$ & $-158.86 \pm 0.51$ & $+0.58 \pm 0.59$ \\
\hline R436Xc & $+147.76 \pm 4.16$ & $+167.51 \pm 0.37$ & $+157.64 \pm 4.18$ & $+142.47 \pm 1.94$ & $+152.48 \pm 0.37$ & $+147.48 \pm 1.97$ & $+10.16 \pm 4.62$ \\
\hline Y447H & $-3.92 \pm 2.48$ & $+3.06 \pm 0.20$ & $-0.43 \pm 2.49$ & $+1.72 \pm 0.07$ & $+2.28 \pm 0.17$ & $+2.00 \pm 0.18$ & $-2.43 \pm 2.49$ \\
\hline $\mathrm{T} 450 \mathrm{~N}$ & $-56.55 \pm 0.26$ & $-57.95 \pm 0.36$ & $-57.25 \pm 0.44$ & $-57.54 \pm 0.49$ & $-56.09 \pm 0.04$ & $-56.82 \pm 0.40$ & $-0.44 \pm 0.60$ \\
\hline N461S & $+80.98 \pm 0.22$ & $+80.71 \pm 0.39$ & $+80.85 \pm 0.45$ & $+74.43 \pm 0.10$ & $+75.39 \pm 0.11$ & $+74.91 \pm 0.15$ & $+5.94 \pm 0.47$ \\
\hline R464C & $+183.94 \pm 0.26$ & $+184.36 \pm 0.69$ & $+184.15 \pm 0.74$ & $+173.08 \pm 0.12$ & $+173.76 \pm 0.12$ & $+173.42 \pm 0.17$ & $+10.73 \pm 0.76$ \\
\hline R464Xc & $+165.96 \pm 0.65$ & $+163.76 \pm 0.47$ & $+164.86 \pm 0.80$ & $+148.66 \pm 2.39$ & $+155.63 \pm 0.22$ & $+152.15 \pm 2.42$ & $+12.72 \pm 2.55$ \\
\hline A476T & $-29.77 \pm 0.12$ & $-29.04 \pm 0.10$ & $-29.41 \pm 0.16$ & $-30.23 \pm 0.07$ & $-29.03 \pm 0.07$ & $-29.63 \pm 0.10$ & $+0.23 \pm 0.18$ \\
\hline A476V & $-18.83 \pm 0.10$ & $-18.21 \pm 0.14$ & $-18.52 \pm 0.17$ & $-18.62 \pm 0.05$ & $-17.62 \pm 0.24$ & $-18.12 \pm 0.25$ & $-0.40 \pm 0.30$ \\
\hline R482C & $+171.60 \pm 0.42$ & $+173.09 \pm 0.41$ & $+172.35 \pm 0.59$ & $+171.21 \pm 0.09$ & $+172.21 \pm 0.08$ & $+171.71 \pm 0.12$ & $+0.63 \pm 0.60$ \\
\hline D489Y & $+68.79 \pm 1.55$ & $+74.50 \pm 0.54$ & $+71.65 \pm 1.64$ & $+67.49 \pm 0.74$ & $+72.16 \pm 0.74$ & $+69.83 \pm 1.05$ & $+1.82 \pm 1.95$ \\
\hline R492G & $+164.21 \pm 0.70$ & $+162.60 \pm 0.38$ & $+163.41 \pm 0.80$ & $+160.21 \pm 0.21$ & $+160.93 \pm 0.12$ & $+160.57 \pm 0.24$ & $+2.84 \pm 0.83$ \\
\hline R495G & $+158.19 \pm 0.40$ & $+157.59 \pm 0.21$ & $+157.89 \pm 0.45$ & $+157.40 \pm 0.19$ & $+158.34 \pm 0.28$ & $+157.87 \pm 0.34$ & $+0.02 \pm 0.56$ \\
\hline
\end{tabular}

a $4434 \mathrm{P}$ and P474L cannot be examined by TI and hence do not appear in this table. ${ }^{b}$ Performed in "reverse" to other cases, i.e., $\lambda=0$ for mutant, $\lambda=1$ for wildtype. See Methods for more details.

"ProPKA prediction assigns this case has HIE, indicated as X in the one-letter notation of $p m x$. See Methods for more details. 
Table S4. Energetic data determined by thermodynamic integration for ZBTB18 variants bound to Rnd2-E2. ${ }^{\text {a }}$

\begin{tabular}{|c|c|c|c|c|c|c|c|}
\hline \multirow{2}{*}{$\begin{array}{l}\text { ZBTB18 } \\
\text { Variant }\end{array}$} & \multicolumn{3}{|c|}{ Bound } & \multicolumn{3}{|c|}{ Unbound } & \multirow{2}{*}{$\begin{array}{c}\Delta \Delta \mathrm{G} \\
(\mathrm{kcal} / \mathrm{mol})\end{array}$} \\
\hline & $\begin{array}{l}\Delta \text { G Forward } \\
\text { (kcal/mol) }\end{array}$ & $\begin{array}{c}\Delta \mathrm{G} \text { Reverse } \\
\text { (kcal/mol) }\end{array}$ & $\begin{array}{l}\Delta \mathrm{G} \text { Average } \\
\text { (kcal/mol) }\end{array}$ & $\begin{array}{c}\Delta G \text { Forward } \\
\text { (kcal/mol) }\end{array}$ & $\begin{array}{c}\Delta \mathrm{G} \text { Reverse } \\
\text { (kcal/mol) }\end{array}$ & $\begin{array}{c}\Delta \mathrm{G} \text { Average } \\
\text { (kcal/mol) }\end{array}$ & \\
\hline L398V & $+1.22 \pm 0.35$ & $+2.61 \pm 0.35$ & $+1.92 \pm 0.36$ & $+1.09 \pm 0.39$ & $+3.04 \pm 0.33$ & $+2.07 \pm 0.51$ & $-0.15 \pm 0.63$ \\
\hline T400Mb & $+31.38 \pm 0.45$ & $+30.72 \pm 0.45$ & $+31.05 \pm 0.77$ & $+30.32 \pm 0.20$ & $+31.65 \pm 0.79$ & $+30.99 \pm 0.81$ & $+0.06 \pm 1.12$ \\
\hline R403C & $+179.19 \pm 0.3$ & $+180.67 \pm 0.30$ & $+179.93 \pm 0.75$ & $+174.91 \pm 0.11$ & $+176.74 \pm 0.26$ & $+175.83 \pm 0.28$ & $+4.11 \pm 0.80$ \\
\hline R409C & $+173.47 \pm 0.14$ & $+173.61 \pm 0.14$ & $+173.54 \pm 0.24$ & $+172.41 \pm 0.35$ & $+174.54 \pm 0.24$ & $+173.48 \pm 0.42$ & $+0.07 \pm 0.49$ \\
\hline A414T & $-28.95 \pm 0.14$ & $-27.31 \pm 0.14$ & $-28.13 \pm 0.21$ & $-29.58 \pm 0.06$ & $-27.27 \pm 0.19$ & $-28.43 \pm 0.20$ & $+0.29 \pm 0.29$ \\
\hline S422L & $-7.55 \pm 0.18$ & $-5.96 \pm 0.18$ & $-6.76 \pm 0.24$ & $-7.12 \pm 0.11$ & $-7.69 \pm 0.11$ & $-7.41 \pm 0.16$ & $+0.65 \pm 0.29$ \\
\hline F428C & $+1.07 \pm 0.2$ & $+2.47 \pm 0.20$ & $+1.77 \pm 0.24$ & $-1.84 \pm 0.15$ & $+0.97 \pm 0.19$ & $-0.44 \pm 0.24$ & $+2.21 \pm 0.34$ \\
\hline K435R & $-159.85 \pm 0.46$ & $-158.57 \pm+0.46$ & $-159.21 \pm 0.47$ & $-159.78 \pm 0.50$ & $-157.93 \pm 0.10$ & $-158.86 \pm 0.51$ & $-0.35 \pm 0.69$ \\
\hline R436Xc & $+154.67 \pm 0.4$ & $+156.60 \pm 0.60$ & $+155.64 \pm 0.60$ & $+142.47 \pm 1.94$ & $+152.48 \pm 0.37$ & $+147.48 \pm 1.97$ & $+8.16 \pm 2.10$ \\
\hline Y447H & $+1.61 \pm 0.32$ & $+3.55 \pm 0.32$ & $+2.58 \pm 0.40$ & $+1.72 \pm 0.07$ & $+2.28 \pm 0.17$ & $+2.00 \pm 0.18$ & $+0.58 \pm 0.44$ \\
\hline T450N & $-56.18 \pm 0.07$ & $-56.02 \pm 0.07$ & $-56.10 \pm 0.11$ & $-57.54 \pm 0.49$ & $-56.09 \pm 0.04$ & $-56.82 \pm 0.40$ & $+0.71 \pm 0.42$ \\
\hline N461S & $+76.78 \pm 0.33$ & $+78.6 \pm 0.33$ & $+77.69 \pm 0.68$ & $+74.43 \pm 0.10$ & $+75.39 \pm 0.11$ & $+74.91 \pm 0.15$ & $+2.78 \pm 0.69$ \\
\hline R464C & $+179.67 \pm 0.25$ & $+180.72 \pm 0.25$ & $+180.20 \pm 1.44$ & $+173.08 \pm 0.12$ & $+173.76 \pm 0.12$ & $+173.42 \pm 0.17$ & $+6.77 \pm 1.45$ \\
\hline R464Xc & $+161.53 \pm 0.47$ & $+159.6 \pm 0.47$ & $+8.42 \pm 2.58$ & $+148.66 \pm 2.39$ & $+155.63 \pm 0.22$ & $+152.15 \pm 2.42$ & $+8.42 \pm 2.58$ \\
\hline A476T & $-29.44 \pm 0.15$ & $-28.31 \pm 0.15$ & $-28.88 \pm 0.20$ & $-30.23 \pm 0.07$ & $-29.03 \pm 0.07$ & $-29.63 \pm 0.10$ & $+0.76 \pm 0.22$ \\
\hline A476V & $-18.53 \pm 0.1$ & $-16.57 \pm 0.10$ & $-17.55 \pm 0.20$ & $-18.62 \pm 0.05$ & $-17.62 \pm 0.24$ & $-18.12 \pm 0.25$ & $+0.57 \pm 0.31$ \\
\hline R482C & $+172.08 \pm 0.4$ & $+174.44 \pm 0.40$ & $+173.26 \pm 0.66$ & $+171.21 \pm 0.09$ & $+172.21 \pm 0.08$ & $+\mathbf{1 7 1 . 7 1} \pm \mathbf{0 . 1 2}$ & $+1.55 \pm 0.67$ \\
\hline D489Y & $+69.66 \pm 0.8$ & $+73.11 \pm 0.80$ & $+71.39 \pm 0.96$ & $+67.49 \pm 0.74$ & $+72.16 \pm 0.74$ & $+69.83 \pm 1.05$ & $+1.56 \pm 1.42$ \\
\hline R492G & $+163.55 \pm 0.27$ & $+164.91 \pm 0.27$ & $+164.23 \pm 0.67$ & $+160.21 \pm 0.21$ & $+160.93 \pm 0.12$ & $+160.57 \pm 0.24$ & $+3.66 \pm 0.71$ \\
\hline R495G & $+157.7 \pm 0.33$ & $+159.06 \pm 0.33$ & $+158.38 \pm 0.53$ & $+157.40 \pm 0.19$ & $+158.34 \pm 0.28$ & $+157.87 \pm 0.34$ & $+0.51 \pm 0.63$ \\
\hline
\end{tabular}

${ }^{\mathrm{a}} \mathrm{L} 434 \mathrm{P}$ and P474L cannot be examined by TI and hence do not appear in this table. ${ }^{\mathrm{b}}$ Performed in "reverse" to other cases, i.e., $\lambda=0$ for mutant, $\lambda=1$ for wildtype. See Methods for more details. "ProPKA prediction assigns this case has HIE, indicated as X in the one-letter notation of pmx. See Methods for more details. 
Table S5. Energetic data determined by thermodynamic integration for ZBTB18 variants bound to Id2-Bs1. ${ }^{\text {a }}$

\begin{tabular}{|c|c|c|c|c|c|c|c|}
\hline \multirow{2}{*}{$\begin{array}{l}\text { ZBTB18 } \\
\text { Variant }\end{array}$} & \multicolumn{3}{|c|}{ Bound } & \multicolumn{3}{|c|}{ Unbound } & \multirow{2}{*}{$\begin{array}{c}\Delta \Delta \mathrm{G} \\
\mathrm{kcal} / \mathrm{mol})\end{array}$} \\
\hline & $\begin{array}{c}\Delta \text { G Forward } \\
\text { (kcal/mol) }\end{array}$ & $\begin{array}{c}\Delta \mathrm{G} \text { Reverse } \\
(\mathrm{kcal} / \mathrm{mol})\end{array}$ & $\begin{array}{c}\Delta \text { G Average } \\
\text { (kcal/mol) }\end{array}$ & $\begin{array}{c}\Delta \text { G Forward } \\
\text { (kcal/mol) }\end{array}$ & $\begin{array}{l}\Delta \text { G Reverse } \\
\text { (kcal/mol) }\end{array}$ & $\begin{array}{l}\Delta \mathrm{G} \text { Average } \\
\text { (kcal/mol) }\end{array}$ & \\
\hline L398V & $+2.03 \pm 0.11$ & $+2.69 \pm 0.08$ & $+2.36 \pm 0.14$ & $+1.09 \pm 0.39$ & $+3.04 \pm 0.33$ & $+2.07 \pm 0.51$ & $+0.30 \pm 0.53$ \\
\hline T400Mb & $+33.29 \pm 0.27$ & $+29.28 \pm 0.39$ & $+31.29 \pm 0.47$ & $+30.32 \pm 0.20$ & $+31.65 \pm 0.79$ & $+30.99 \pm 0.81$ & $+0.30 \pm 0.94$ \\
\hline R403C & $+179.27 \pm 0.79$ & $+180.95 \pm 1.1$ & $+180.11 \pm 1.35$ & $+174.91 \pm 0.11$ & $+176.74 \pm 0.26$ & $+175.83 \pm 0.28$ & $+4.29 \pm 1.38$ \\
\hline R409C & $+174.73 \pm 0.39$ & $+175.97 \pm 0.47$ & $+175.35 \pm 0.61$ & $+172.41 \pm 0.35$ & $+174.54 \pm 0.24$ & $+173.48 \pm 0.42$ & $+1.88 \pm 0.74$ \\
\hline A414T & $-28.64 \pm 0.12$ & $-27.61 \pm 0.1$ & $-28.13 \pm 0.16$ & $-29.58 \pm 0.06$ & $-27.27 \pm 0.19$ & $-28.43 \pm 0.20$ & $+0.30 \pm 0.25$ \\
\hline S422L & $-7.18 \pm 0.13$ & $-5.72 \pm 0.14$ & $-6.45 \pm 0.19$ & $-7.12 \pm 0.11$ & $-7.69 \pm 0.11$ & $-7.41 \pm 0.16$ & $+0.96 \pm 0.25$ \\
\hline F428C & $+0.79 \pm 0.2$ & $+8.43 \pm 2.77$ & $+4.61 \pm 2.78$ & $-1.84 \pm 0.15$ & $+0.97 \pm 0.19$ & $-0.44 \pm 0.24$ & $+5.05 \pm 2.79$ \\
\hline K435R & $-158.41 \pm 0.18$ & $-157.99 \pm 0.17$ & $-158.20 \pm 0.25$ & $-159.78 \pm 0.50$ & $-157.93 \pm 0.10$ & $-158.86 \pm 0.51$ & $+0.66 \pm 0.57$ \\
\hline R436Xc & $+157.48 \pm 0.54$ & $+158.42 \pm 0.88$ & $+157.95 \pm 1.03$ & $+142.47 \pm 1.94$ & $+152.48 \pm 0.37$ & $+147.48 \pm 1.97$ & $+10.48 \pm 2.23$ \\
\hline Y447H & $+1.72 \pm 0.09$ & $+2.90 \pm 0.27$ & $+2.31 \pm 0.28$ & $+1.72 \pm 0.07$ & $+2.28 \pm 0.17$ & $+2.00 \pm 0.18$ & $+0.31 \pm 0.34$ \\
\hline $\mathrm{T} 450 \mathrm{~N}$ & $-56.23 \pm 0.08$ & $-56.21 \pm 0.06$ & $-56.22 \pm 0.10$ & $-57.54 \pm 0.49$ & $-56.09 \pm 0.04$ & $-56.82 \pm 0.40$ & $+0.59 \pm 0.41$ \\
\hline $\mathbf{N} 461 \mathrm{~S}$ & $+75.57 \pm 0.2$ & $+77.96 \pm 0.82$ & $+76.77 \pm 0.84$ & $+74.43 \pm 0.10$ & $+75.39 \pm 0.11$ & $+74.91 \pm 0.15$ & $+1.85 \pm 0.86$ \\
\hline R464C & $+179.86 \pm 0.34$ & $+179.96 \pm 0.86$ & $+179.91 \pm 0.92$ & $+173.08 \pm 0.12$ & $+173.76 \pm 0.12$ & $+173.42 \pm 0.17$ & $+6.49 \pm 0.94$ \\
\hline $\mathrm{R} 464 \mathrm{Xc}$ & $+161.86 \pm 0.46$ & $+158.01 \pm 0.23$ & $+159.94 \pm 0.51$ & $+148.66 \pm 2.39$ & $+155.63 \pm 0.22$ & $+152.15 \pm 2.42$ & $+7.79 \pm 2.47$ \\
\hline A476T & $-28.28 \pm 0.16$ & $-27.07 \pm 0.1$ & $-27.68 \pm 0.19$ & $-30.23 \pm 0.07$ & $-29.03 \pm 0.07$ & $-29.63 \pm 0.10$ & $+1.96 \pm 0.21$ \\
\hline A476V & $-17.16 \pm 0.2$ & $-14.36 \pm 0.63$ & $-15.76 \pm 0.66$ & $-18.62 \pm 0.05$ & $-17.62 \pm 0.24$ & $-18.12 \pm 0.25$ & $+2.36 \pm 0.70$ \\
\hline R482C & $+172.35 \pm 0.36$ & $+173.37 \pm 0.22$ & $+172.86 \pm 0.42$ & $+171.21 \pm 0.09$ & $+172.21 \pm 0.08$ & $+171.71 \pm 0.12$ & $+1.15 \pm 0.44$ \\
\hline D489Y & $+75.39 \pm 0.39$ & $+76.7 \pm 0.53$ & $+76.05 \pm 0.66$ & $+67.49 \pm 0.74$ & $+72.16 \pm 0.74$ & $+69.83 \pm 1.05$ & $+6.22 \pm 1.24$ \\
\hline $\mathbf{R 4 9 2 G}$ & $+166.89 \pm 0.69$ & $+164.33 \pm 0.78$ & $+165.61 \pm 1.04$ & $+160.21 \pm 0.21$ & $+160.93 \pm 0.12$ & $+160.57 \pm 0.24$ & $+5.04 \pm 1.07$ \\
\hline R495G & $+159.05 \pm 0.18$ & $+158.33 \pm 0.19$ & $+158.69 \pm 0.26$ & $+157.40 \pm 0.19$ & $+158.34 \pm 0.28$ & $+157.87 \pm 0.34$ & $+0.82 \pm 0.43$ \\
\hline
\end{tabular}

${ }^{\mathrm{a}} \mathrm{L} 434 \mathrm{P}$ and P474L cannot be examined by TI and hence do not appear in this table. ${ }^{\mathrm{b}}$ Performed in "reverse" to other cases, i.e., $\lambda=0$ for mutant, $\lambda=1$ for wildtype. See Methods for more details. "ProPKA prediction assigns this case has HIE, indicated as X in the one-letter notation of pmx. See Methods for more details. 
Table S6. Energetic data determined by thermodynamic integration for ZBTB18 variants bound to Id2-Bs2. ${ }^{\text {a }}$

\begin{tabular}{|c|c|c|c|c|c|c|c|}
\hline \multirow{2}{*}{$\begin{array}{l}\text { ZBTB18 } \\
\text { Variant }\end{array}$} & \multicolumn{3}{|c|}{ Bound } & \multicolumn{3}{|c|}{ Unbound } & \multirow{2}{*}{$\begin{array}{c}\Delta \Delta \mathrm{G} \\
(\mathrm{kcal} / \mathrm{mol})\end{array}$} \\
\hline & $\begin{array}{c}\Delta \text { G Forward } \\
\text { (kcal/mol) }\end{array}$ & $\begin{array}{l}\Delta \text { G Reverse } \\
\text { (kcal/mol) }\end{array}$ & $\begin{array}{c}\Delta \mathrm{G} \text { Average } \\
\text { (kcal/mol) }\end{array}$ & $\begin{array}{c}\Delta \text { G Forward } \\
\text { (kcal/mol) }\end{array}$ & $\begin{array}{c}\Delta \mathrm{G} \text { Reverse } \\
\text { (kcal/mol) }\end{array}$ & $\begin{array}{l}\Delta \text { G Average } \\
\text { (kcal/mol) }\end{array}$ & \\
\hline L398V & $+1.75 \pm 0.17$ & $+2.73 \pm 0.07$ & $+2.24 \pm 0.18$ & $+1.09 \pm 0.39$ & $+3.04 \pm 0.33$ & $+2.07 \pm 0.51$ & $+0.18 \pm 0.54$ \\
\hline T400Mb & $+33.66 \pm 0.17$ & $+28.54 \pm 0.16$ & $+31.10 \pm 0.23$ & $+30.32 \pm 0.20$ & $+31.65 \pm 0.79$ & $+30.99 \pm 0.81$ & $+0.11 \pm 0.85$ \\
\hline R403C & $+178.93 \pm 0.32$ & $+178.99 \pm 0.99$ & $+178.96 \pm 1.04$ & $+174.91 \pm 0.11$ & $+176.74 \pm 0.26$ & $+175.83 \pm 0.28$ & $+3.14 \pm 1.08$ \\
\hline R409C & $+175.26 \pm 0.29$ & $+177.52 \pm 1.64$ & $+176.39 \pm 1.67$ & $+172.41 \pm 0.35$ & $+174.54 \pm 0.24$ & $+173.48 \pm 0.42$ & $+2.91 \pm 1.72$ \\
\hline A414T & $-29.92 \pm 0.37$ & $-27.24 \pm 0.17$ & $-28.58 \pm 0.41$ & $-29.58 \pm 0.06$ & $-27.27 \pm 0.19$ & $-28.43 \pm 0.20$ & $-0.16 \pm 0.45$ \\
\hline S422L & $-7.24 \pm 0.18$ & $-5.82 \pm 0.11$ & $-6.53 \pm 0.21$ & $-7.12 \pm 0.11$ & $-7.69 \pm 0.11$ & $-7.41 \pm 0.16$ & $+0.88 \pm 0.26$ \\
\hline F428C & $+0.37 \pm 0.15$ & $+2.74 \pm 0.18$ & $+1.56 \pm 0.23$ & $-1.84 \pm 0.15$ & $+0.97 \pm 0.19$ & $-0.44 \pm 0.24$ & $+1.99 \pm 0.34$ \\
\hline K435R & $-158.59 \pm 0.22$ & $-157.33 \pm 0.25$ & $-157.96 \pm 0.33$ & $-159.78 \pm 0.50$ & $-157.93 \pm 0.10$ & $-158.86 \pm 0.51$ & $+0.90 \pm 0.61$ \\
\hline R436Xc & $+158.42 \pm 0.4$ & $162.34 \pm 3.06$ & $+160.38 \pm 3.09$ & $+142.47 \pm 1.94$ & $+152.48 \pm 0.37$ & $+147.48 \pm 1.97$ & $+12.91 \pm 3.66$ \\
\hline Y447H & $+1.05 \pm 0.28$ & $+2.59 \pm 0.12$ & $+1.82 \pm 0.30$ & $+1.72 \pm 0.07$ & $+2.28 \pm 0.17$ & $+2.00 \pm 0.18$ & $-0.18 \pm 0.36$ \\
\hline T450N & $-56.43 \pm 0.14$ & $-56.08 \pm 0.06$ & $-56.26 \pm 0.15$ & $-57.54 \pm 0.49$ & $-56.09 \pm 0.04$ & $-56.82 \pm 0.40$ & $+0.56 \pm 0.43$ \\
\hline N461S & $+77.1 \pm 0.25$ & $+77.18 \pm 0.4$ & $+77.14 \pm 0.47$ & $+74.43 \pm 0.10$ & $+75.39 \pm 0.11$ & $+74.91 \pm 0.15$ & $+2.23 \pm 0.49$ \\
\hline $\mathrm{R} 464 \mathrm{C}$ & $+180.75 \pm 0.35$ & $+180.86 \pm 0.81$ & $+180.81 \pm 0.88$ & $+173.08 \pm 0.12$ & $+173.76 \pm 0.12$ & $+173.42 \pm 0.17$ & $+7.38 \pm 0.90$ \\
\hline $\mathrm{R} 464 \mathrm{Xc}^{\mathrm{c}}$ & $+165.16 \pm 0.43$ & $+160.95 \pm 0.55$ & $+163.06 \pm 0.70$ & $+148.66 \pm 2.39$ & $+155.63 \pm 0.22$ & $+152.15 \pm 2.42$ & $+10.91 \pm 2.52$ \\
\hline A476T & $-29.5 \pm 0.14$ & $-28.13 \pm 0.12$ & $-28.82 \pm 0.18$ & $-30.23 \pm 0.07$ & $-29.03 \pm 0.07$ & $-29.63 \pm 0.10$ & $+0.82 \pm 0.21$ \\
\hline A476V & $-18.38 \pm 0.11$ & $-17.03 \pm 0.14$ & $-17.71 \pm 0.18$ & $-18.62 \pm 0.05$ & $-17.62 \pm 0.24$ & $-18.12 \pm 0.25$ & $+0.42 \pm 0.30$ \\
\hline R482C & $+175.62 \pm 0.25$ & $+173.97 \pm 0.23$ & $+174.80 \pm 0.34$ & $+171.21 \pm 0.09$ & $+172.21 \pm 0.08$ & $+171.71 \pm 0.12$ & $+3.09 \pm 0.36$ \\
\hline D489Y & $+72.41 \pm 0.65$ & $+75.2 \pm 0.26$ & $+73.81 \pm 0.70$ & $+67.49 \pm 0.74$ & $+72.16 \pm 0.74$ & $+69.83 \pm 1.05$ & $+3.98 \pm 1.26$ \\
\hline $\mathbf{R 4 9 2 G}$ & $+168.17 \pm 0.33$ & $+162.59 \pm 0.39$ & $+165.38 \pm 0.51$ & $+160.21 \pm 0.21$ & $+160.93 \pm 0.12$ & $+160.57 \pm 0.24$ & $+4.81 \pm 0.57$ \\
\hline R495G & $+158.31 \pm 0.15$ & $+158.76 \pm 0.33$ & $+158.54 \pm 0.36$ & $+157.40 \pm 0.19$ & $+158.34 \pm 0.28$ & $+157.87 \pm 0.34$ & $+0.66 \pm 0.50$ \\
\hline
\end{tabular}

${ }^{\mathrm{a}} \mathrm{L} 434 \mathrm{P}$ and P474L cannot be examined by TI and hence do not appear in this table. ${ }^{\mathrm{b}}$ Performed in "reverse" to other cases, i.e., $\lambda=0$ for mutant, $\lambda=1$ for wildtype. See Methods for more details. "ProPKA prediction assigns this case has HIE, indicated as X in the one-letter notation of pmx. See Methods for more details. 
Table S7. Relative binding energies (relative to wildtype) determined by MM-GB/SA for ZBTB18 variant binding to DNA fragments. ${ }^{\mathrm{a}}$

\begin{tabular}{|c|c|c|c|c|c|c|c|c|}
\hline & \multicolumn{2}{|c|}{ Rnd2-E1 } & \multicolumn{2}{|c|}{ Rnd2-E2 } & \multicolumn{2}{|c|}{$I d 2-B s 1$} & \multicolumn{2}{|c|}{$I d 2-B s 2$} \\
\hline DNA & $\Delta \mathrm{G}_{\text {bind }}$ & $\Delta \Delta \mathrm{G}$ & $\Delta \mathrm{G}_{\text {bind }}$ & $\Delta \Delta \mathrm{G}$ & $\Delta \mathrm{G}_{\text {bind }}$ & $\Delta \Delta \mathrm{G}$ & $\Delta \mathrm{G}_{\text {bind }}$ & $\Delta \Delta \mathrm{G}$ \\
\hline WT & $-119.8 \pm 0.3$ & $N / A$ & $-125.2 \pm 0.3$ & $N / A$ & $-96.7 \pm 0.3$ & $N / A$ & $-107.0 \pm 0.4$ & $N / A$ \\
\hline L398V & $-113.8 \pm 0.3$ & $+6.0 \pm 0.4$ & $-133.3 \pm 0.3$ & $-8.1 \pm 0.4$ & $-96.9 \pm 0.5$ & $-0.2 \pm 0.6$ & $-117.4 \pm 0.9$ & $-10.4 \pm 1.0$ \\
\hline T400M & $-134.9 \pm 0.5$ & $-15.1 \pm 0.6$ & $-120.2 \pm 0.3$ & $+5.0 \pm 0.4$ & $-94.2 \pm 0.4$ & $+2.5 \pm 0.5$ & $-125.2 \pm 0.8$ & $-18.2 \pm 0.9$ \\
\hline R403C & $-125.2 \pm 0.2$ & $-5.4 \pm 0.4$ & $-111.3 \pm 0.4$ & $+13.9 \pm 0.5$ & $-119.2 \pm 0.3$ & $-22.5 \pm 0.4$ & $-138.6 \pm 0.5$ & $-31.6 \pm 0.6$ \\
\hline R409C & $-98.9 \pm 0.7$ & $+20.9 \pm 0.8$ & $-143.1 \pm 0.4$ & $-17.9 \pm 0.5$ & $-129.2 \pm 0.3$ & $-32.5 \pm 0.4$ & $-115.9 \pm 0.3$ & $-8.9 \pm 0.5$ \\
\hline A414T & $-124.2 \pm 0.4$ & $-4.4 \pm 0.5$ & $-153.1 \pm 0.3$ & $-27.9 \pm 0.4$ & $-81.2 \pm 0.4$ & $+15.5 \pm 0.5$ & $-123.1 \pm 0.3$ & $-16.1 \pm 0.5$ \\
\hline S422L & $-131.3 \pm 0.4$ & $-11.5 \pm 0.5$ & $-126.4 \pm 0.3$ & $-1.2 \pm 0.4$ & $-101.2 \pm 0.4$ & $-4.5 \pm 0.5$ & $-125.5 \pm 0.8$ & $-18.5 \pm 0.9$ \\
\hline F428C & $-146.1 \pm 0.4$ & $-26.3 \pm 0.5$ & $-124.8 \pm 0.6$ & $+0.4 \pm 0.7$ & $-131.9 \pm 0.3$ & $-35.2 \pm 0.4$ & $-122.5 \pm 0.3$ & $-15.5 \pm 0.5$ \\
\hline L434P & $-117.3 \pm 0.3$ & $+2.5 \pm 0.4$ & NSC & NSC & $-114.5 \pm 0.3$ & $-17.8 \pm 0.4$ & $-114.8 \pm 0.5$ & $-7.8 \pm 0.6$ \\
\hline K435R & $-143.1 \pm 0.4$ & $-23.3 \pm 0.5$ & $-135.7 \pm 0.3$ & $-10.5 \pm 0.4$ & $-74.6 \pm 0.3$ & $+22.1 \pm 0.4$ & $-136.3 \pm 0.3$ & $-29.3 \pm 0.5$ \\
\hline R436H & $-103.6 \pm 0.4$ & $+16.2 \pm 0.5$ & $-133.2 \pm 0.3$ & $-8.0 \pm 0.4$ & NSC & NSC & $-112.4 \pm 0.3$ & $-5.4 \pm 0.5$ \\
\hline Y447H & $-108.6 \pm 0.3$ & $+11.2 \pm 0.4$ & $-120.3 \pm 0.4$ & $+4.9 \pm 0.5$ & NSC & NSC & $-122.9 \pm 0.4$ & $-15.9 \pm 0.6$ \\
\hline T450N & $-120.5 \pm 0.5$ & $-0.7 \pm 0.6$ & $-131.7 \pm 0.3$ & $-6.5 \pm 0.4$ & NSC & NSC & $-135.7 \pm 0.5$ & $-28.7 \pm 0.6$ \\
\hline N461S & $-129.6 \pm 0.3$ & $-9.8 \pm 0.4$ & $-108.1 \pm 0.3$ & $+17.1 \pm 0.4$ & $-129.6 \pm 0.5$ & $-32.9 \pm 0.6$ & NSC & NSC \\
\hline R464C & $-106.9 \pm 0.2$ & $+12.9 \pm 0.4$ & $-94.3 \pm 0.4$ & $+30.9 \pm 0.5$ & $-43.7 \pm 0.4$ & $+53.0 \pm 0.5$ & $-121.9 \pm 0.3$ & $-14.9 \pm 0.5$ \\
\hline R464H & $-112.0 \pm 0.4$ & $+7.8 \pm 0.5$ & $-99.1 \pm 0.3$ & $+26.1 \pm 0.4$ & NSC & NSC & $-77.3 \pm 0.8$ & $+29.7 \pm 0.9$ \\
\hline P474L & $-126.7 \pm 0.4$ & $-6.9 \pm 0.5$ & $-138.6 \pm 0.3$ & $-13.4 \pm 0.4$ & $-126.0 \pm 0.3$ & $-29.3 \pm 0.4$ & $-144.3 \pm 0.4$ & $-37.3 \pm 0.6$ \\
\hline A476T & $-111.8 \pm 0.3$ & $+8.0 \pm 0.4$ & $-137.3 \pm 0.3$ & $-12.1 \pm 0.4$ & $-99.1 \pm 0.3$ & $-2.4 \pm 0.4$ & $-100.7 \pm 0.4$ & $+6.3 \pm 0.6$ \\
\hline A476V & $-119.3 \pm 0.4$ & $+0.5 \pm 0.5$ & $-121.6 \pm 0.4$ & $+3.6 \pm 0.5$ & $-104.7 \pm 0.4$ & $-8.0 \pm 0.5$ & $-140.0 \pm 0.3$ & $-33.0 \pm 0.5$ \\
\hline R482C & $-119.0 \pm 0.4$ & $+0.8 \pm 0.5$ & $-119.6 \pm 0.3$ & $+5.6 \pm 0.4$ & $-90.4 \pm 0.4$ & $+6.3 \pm 0.5$ & $-127.7 \pm 0.4$ & $-20.7 \pm 0.6$ \\
\hline D489Y & $-122.1 \pm 0.4$ & $-2.3 \pm 0.5$ & $-107.9 \pm 0.3$ & $+17.3 \pm 0.4$ & $-120.4 \pm 0.3$ & $-23.7 \pm 0.4$ & $-131.2 \pm 0.5$ & $-24.2 \pm 0.6$ \\
\hline R492C & $-114.5 \pm 0.5$ & $+5.3 \pm 0.6$ & $-122.8 \pm 0.3$ & $+2.4 \pm 0.4$ & $-108.8 \pm 0.3$ & $-12.1 \pm 0.4$ & $-110.1 \pm 0.3$ & $-3.1 \pm 0.5$ \\
\hline R495G & $-126.4 \pm 0.3$ & $-6.6 \pm 0.4$ & $-126.9 \pm 0.4$ & $-1.7 \pm 0.5$ & NSC & NSC & $-98.5 \pm 0.3$ & $+8.5 \pm 0.5$ \\
\hline
\end{tabular}

${ }^{a} \mathrm{NSC}$ in table indicates non-stable complex; binding energies were not calculated by MM-GB/SA due to an insufficient number of sufficiently stable simulations being obtained for this complex. See Hemming et al. 2019, Human Mutation, v40, p1841-1855 and Hemming et al. 2020, Human Mutation, v41, p1629-1644 for further details. 
Table S8. Relative binding energies (relative to wildtype) determined by Schrodinger Residue Scanning for ZBTB18 variant binding to DNA fragments.

\begin{tabular}{|l|c|c|c|c|}
\hline ZBTB18 & $R n d 2-E 1$ & $R n d 2-E 2$ & $I d 2-B s 1$ & $I d 2-B s 2$ \\
\cline { 2 - 5 } Variant & $\Delta \Delta \mathrm{G}$ & $\Delta \Delta \mathrm{G}$ & $\Delta \Delta \mathrm{G}$ & $\Delta \Delta \mathrm{G}$ \\
\hline L398V & -0.25 & -0.17 & -0.35 & -0.22 \\
\cline { 2 - 5 } T400M & +10.11 & +11.81 & +12.27 & +12.2 \\
\cline { 2 - 5 } R403C & +4.21 & +5.05 & +4.96 & +4.98 \\
\hline R409C & +4.17 & +4.11 & +4.19 & +4.2 \\
\hline A414T & -0.05 & -0.04 & -0.05 & -0.06 \\
\hline S422L & -0.03 & -0.29 & -0.21 & -0.16 \\
\hline F428C & -7.45 & -7.75 & -7.72 & -7.72 \\
\hline L434P & -2.12 & -2.57 & -2.34 & -2.52 \\
\hline K435R & -1.03 & -2.95 & -2.14 & -3.37 \\
R436H & +20.24 & +17.97 & +17.97 & +19.32 \\
\hline Y447H & +0.86 & +1.72 & -0.26 & -0.22 \\
\hline T450N & -0.04 & -0.04 & +0.01 & +0.02 \\
\hline N461S & +1.27 & +2.6 & +13.03 & +7.37 \\
\hline R464C & +17.42 & +18.03 & +18.04 & +16.09 \\
\hline R464H & +17.03 & +17.73 & +17.51 & +15.35 \\
\hline P474L & +0.67 & +0.8 & +0.85 & +0.83 \\
\hline A476T & -0.19 & -0.19 & -0.21 & -0.21 \\
\hline A476V & +0.05 & +0.01 & +0.03 & -0.01 \\
\hline R482C & +8.7 & +14.63 & +15.59 & +11.79 \\
\hline D489Y & -3.26 & -2.34 & -2.32 & -7.98 \\
\hline R492C & +17.45 & +15.8 & +16.71 & +17.17 \\
\hline R495G & +5.16 & +5.25 & +5.27 & +5.25 \\
\hline
\end{tabular}


Table S9. Relative binding energies (relative to wildtype) determined by mCSM-NA for ZBTB18 variant binding to DNA fragments.

\begin{tabular}{|l|c|c|c|c|}
\hline ZBTB18 & $R n d 2-E 1$ & $R n d 2-E 2$ & $I d 2-B s 1$ & $I d 2-B s 2$ \\
\cline { 2 - 5 } Variant & $\Delta \Delta \mathrm{G}$ & $\Delta \Delta \mathrm{G}$ & $\Delta \Delta \mathrm{G}$ & $\Delta \Delta \mathrm{G}$ \\
\hline L398V & +0.006 & -0.444 & +0.342 & +0.092 \\
\cline { 2 - 5 } T400M & +0.446 & -1.090 & +0.894 & +0.432 \\
\cline { 2 - 5 } R403C & +0.618 & -1.008 & +1.006 & +0.620 \\
\hline R409C & +0.834 & -0.822 & +0.814 & +0.824 \\
\hline A414T & -1.304 & +1.308 & -1.334 & -1.302 \\
\hline S422L & +1.386 & -1.406 & +1.402 & +1.404 \\
\hline F428C & +0.492 & -0.326 & +0.480 & +0.390 \\
\hline L434P & -0.154 & +0.338 & -0.018 & +0.050 \\
\hline K435R & -1.148 & +2.006 & -1.832 & -2.310 \\
\hline R436H & +1.972 & -2.012 & +1.988 & +1.824 \\
Y447H & -0.772 & +1.296 & -1.102 & -1.424 \\
\hline T450N & -0.006 & +0.006 & -0.002 & -0.008 \\
\hline N461S & -0.904 & +0.802 & +1.272 & +0.510 \\
\hline R464C & +2.422 & -2.272 & +2.802 & +2.960 \\
\hline R464H & +1.574 & -1.270 & +1.146 & +1.576 \\
\hline P474L & -0.916 & +0.938 & -0.734 & -0.702 \\
\hline A476T & -0.668 & +0.680 & -1.180 & -1.320 \\
\hline A476V & -0.068 & +0.138 & -0.386 & -0.508 \\
\hline R482C & +0.540 & -0.480 & +0.890 & +1.220 \\
\hline D489Y & -1.304 & +0.976 & -1.242 & -1.348 \\
\hline R492C & +4.076 & -3.148 & +4.500 & +5.118 \\
\hline R495G & +1.512 & -0.666 & +1.312 & +2.070 \\
\hline
\end{tabular}


Table S10. Relative binding energies (relative to wildtype) determined by SAMPDI for ZBTB18 variant binding to DNA fragments.

\begin{tabular}{|l|c|c|c|c|}
\hline ZBTB18 & $R n d 2-E 1$ & $R n d 2-E 2$ & $I d 2-B s 1$ & $I d 2-B s 2$ \\
\cline { 2 - 5 } Variant & $\Delta \Delta \mathrm{G}$ & $\Delta \Delta \mathrm{G}$ & $\Delta \Delta \mathrm{G}$ & $\Delta \Delta \mathrm{G}$ \\
\hline L398V & $+0.44 \pm 0.21$ & $+0.52 \pm 0.14$ & $+0.65 \pm 0.16$ & $+0.29 \pm 0.45$ \\
\cline { 2 - 5 } T400M & $+0.32 \pm 0.33$ & $+0.21 \pm 0.32$ & $+0.61 \pm 0.19$ & $+0.20 \pm 0.15$ \\
\cline { 2 - 5 } R403C & $+1.13 \pm 0.18$ & $+0.88 \pm 0.21$ & $+1.13 \pm 0.03$ & $+0.71 \pm 0.25$ \\
\hline R409C & $+1.60 \pm 0.41$ & $+0.99 \pm 0.14$ & $+1.62 \pm 0.12$ & $+1.12 \pm 0.04$ \\
\cline { 2 - 5 } A414T & $+0.45 \pm 0.18$ & $+0.57 \pm 0.15$ & $+0.53 \pm 0.08$ & $+0.18 \pm 0.27$ \\
\hline S422L & $+0.39 \pm 0.02$ & $+0.67 \pm 0.12$ & $+0.49 \pm 0.24$ & $+0.40 \pm 0.24$ \\
\hline F428C & $+1.08 \pm 0.22$ & $+0.94 \pm 0.10$ & $+0.90 \pm 0.20$ & $+0.63 \pm 0.14$ \\
\hline L434P & $+0.17 \pm 0.20$ & $+0.42 \pm 0.26$ & $+0.05 \pm 0.18$ & $-0.09 \pm 0.19$ \\
\hline K435R & $+0.97 \pm 0.37$ & $+0.41 \pm 0.19$ & $+0.77 \pm 0.50$ & $+0.75 \pm 0.26$ \\
\hline R436H & $+0.85 \pm 0.10$ & $+0.91 \pm 0.31$ & $+0.77 \pm 0.27$ & $+1.12 \pm 0.25$ \\
\hline Y447H & $+0.94 \pm 0.09$ & $+0.74 \pm 0.18$ & $+0.67 \pm 0.25$ & $+0.26 \pm 0.24$ \\
\hline T450N & $+0.50 \pm 0.11$ & $+0.87 \pm 0.21$ & $+0.46 \pm 0.07$ & $+0.45 \pm 0.33$ \\
\hline N461S & $+0.56 \pm 0.24$ & $+0.45 \pm 0.35$ & $+0.28 \pm 0.23$ & $+0.40 \pm 0.25$ \\
\hline R464C & $+0.84 \pm 0.24$ & $+0.69 \pm 0.23$ & $+0.15 \pm 0.27$ & $+0.32 \pm 0.32$ \\
\hline R464H & $+1.21 \pm 0.20$ & $+0.89 \pm 0.12$ & $+0.32 \pm 0.16$ & $+0.21 \pm 0.23$ \\
\hline P474L & $+0.97 \pm 0.04$ & $+0.64 \pm 0.31$ & $+0.42 \pm 0.29$ & $+0.66 \pm 0.23$ \\
\hline A476T & $+0.25 \pm 0.09$ & $+0.41 \pm 0.22$ & $+0.58 \pm 0.45$ & $-0.04 \pm 0.35$ \\
\hline A476V & $+0.45 \pm 0.07$ & $+0.92 \pm 0.59$ & $+0.50 \pm 0.04$ & $+0.18 \pm 0.22$ \\
\hline R482C & $+0.88 \pm 0.17$ & $+0.54 \pm 0.16$ & $+1.53 \pm 0.17$ & $+0.70 \pm 0.14$ \\
\hline D489Y & $-0.07 \pm 0.24$ & $-1.11 \pm 0.29$ & $-0.11 \pm 0.31$ & $-0.67 \pm 0.45$ \\
\hline R492C & $+0.33 \pm 0.03$ & $+0.91 \pm 0.15$ & $+0.32 \pm 0.14$ & $+0.59 \pm 0.27$ \\
\hline R495G & $+0.80 \pm 0.32$ & $+0.83 \pm 0.22$ & $+0.81 \pm 0.04$ & $+0.81 \pm 0.46$ \\
\hline
\end{tabular}


Table S11. Energetic data determined by thermodynamic integration for additional ZBTB18 variants.

\begin{tabular}{|c|c|c|c|c|c|c|c|}
\hline \multirow[b]{2}{*}{ Complex } & \multicolumn{3}{|c|}{ Bound } & \multicolumn{3}{|c|}{ Unbound } & \multirow[b]{2}{*}{$\begin{array}{c}\Delta \Delta \mathrm{G} \\
(\mathrm{kcal} / \mathrm{mol})\end{array}$} \\
\hline & $\begin{array}{c}\Delta G \text { Forward } \\
\text { (kcal/mol) }\end{array}$ & $\begin{array}{l}\Delta \text { G Reverse } \\
(\mathrm{kcal} / \mathrm{mol})\end{array}$ & $\begin{array}{l}\Delta \text { G Average } \\
\text { (kcal/mol) }\end{array}$ & $\begin{array}{c}\Delta G \text { Forward } \\
\text { (kcal/mol) }\end{array}$ & $\begin{array}{l}\Delta \text { G Reverse } \\
\text { (kcal/mol) }\end{array}$ & $\begin{array}{l}\Delta \text { G Average } \\
\text { (kcal/mol) }\end{array}$ & \\
\hline $\begin{array}{c}\text { Rnd2-E1 } \\
\text { A413T }\end{array}$ & $-29.16 \pm 0.13$ & $-27.20 \pm 0.22$ & $-28.18 \pm 0.26$ & $-29.33 \pm 0.09$ & $-26.31 \pm 0.44$ & $-27.82 \pm 0.45$ & $-0.36 \pm 0.52$ \\
\hline $\begin{array}{c}\text { Rnd2-E1 } \\
\text { V416I }\end{array}$ & $+20.59 \pm 0.09$ & $+21.39 \pm 0.14$ & $+20.99 \pm 0.17$ & $+20.18 \pm 0.05$ & $+21.02 \pm 0.12$ & $+20.60 \pm 0.13$ & $+0.39 \pm 0.21$ \\
\hline $\begin{array}{c}\text { Rnd2-E1 } \\
\text { R436L }\end{array}$ & $+154.01 \pm 0.24$ & $+170.23 \pm 0.44$ & $+162.12 \pm 0.50$ & $+148.38 \pm 0.13$ & $+149.39 \pm 0.17$ & $+148.89 \pm 0.21$ & $+13.23 \pm 0.54$ \\
\hline $\begin{array}{c}\text { Rnd2-E1 } \\
\text { Y458C }\end{array}$ & $+21.37 \pm 0.55$ & $+26.49 \pm 0.54$ & $+23.93 \pm 0.77$ & $+19.15 \pm 0.09$ & $+22.04 \pm 0.90$ & $+20.60 \pm 0.90$ & $+3.33 \pm 1.19$ \\
\hline $\begin{array}{c}\text { Rnd2-E1 } \\
\text { G488R }\end{array}$ & $-161.08 \pm 0.24$ & $-155.65 \pm 0.72$ & $-158.37 \pm 0.76$ & $-159.62 \pm 0.15$ & $-159.95 \pm 0.11$ & $-159.79 \pm 0.19$ & $+1.42 \pm 0.78$ \\
\hline $\begin{array}{c}\text { Rnd2-E2 } \\
\text { A413T }\end{array}$ & $-29.28 \pm 0.11$ & $-24.29 \pm 0.79$ & $-26.78 \pm 0.80$ & $-29.33 \pm 0.09$ & $-26.31 \pm 0.44$ & $-27.82 \pm 0.45$ & $+1.04 \pm 0.92$ \\
\hline $\begin{array}{c}\text { Rnd2-E2 } \\
\text { V416I }\end{array}$ & $+20.44 \pm 0.11$ & $+21.25 \pm 0.09$ & $+20.85 \pm 0.14$ & $+20.18 \pm 0.05$ & $+21.02 \pm 0.12$ & $+20.60 \pm 0.13$ & $+0.25 \pm 0.19$ \\
\hline $\begin{array}{c}\text { Rnd2-E2 } \\
\text { R436L }\end{array}$ & $+151.46 \pm 0.34$ & $+156.34 \pm 1.07$ & $+153.90 \pm 1.12$ & $+148.38 \pm 0.13$ & $+149.39 \pm 0.17$ & $+148.89 \pm 0.21$ & $+5.01 \pm 1.14$ \\
\hline $\begin{array}{c}\text { Rnd2-E2 } \\
\text { Y458C }\end{array}$ & $+20.29 \pm 0.38$ & $+25.62 \pm 0.93$ & $+22.96 \pm 1.00$ & $+19.15 \pm 0.09$ & $+22.04 \pm 0.90$ & $+20.60 \pm 0.90$ & $+2.36 \pm 1.35$ \\
\hline $\begin{array}{c}\text { Rnd2-E2 } \\
\text { G488R }\end{array}$ & $-161.66 \pm 0.63$ & $-157.81 \pm 0.73$ & $-159.73 \pm 0.96$ & $-159.62 \pm 0.15$ & $-159.95 \pm 0.11$ & $-159.79 \pm 0.19$ & $+0.06 \pm 0.98$ \\
\hline $\begin{array}{c}I d 2-B s 1 \\
\text { A413T }\end{array}$ & $-28.89 \pm 0.12$ & $-24.95 \pm 0.51$ & $-26.92 \pm 0.52$ & $-29.33 \pm 0.09$ & $-26.31 \pm 0.44$ & $-27.82 \pm 0.45$ & $+0.90 \pm 0.69$ \\
\hline $\begin{array}{c}I d 2-B s 1 \\
\text { V416I }\end{array}$ & $+20.06 \pm 0.11$ & $+20.95 \pm 0.11$ & $+20.51 \pm 0.16$ & $+20.18 \pm 0.05$ & $+21.02 \pm 0.12$ & $+20.60 \pm 0.13$ & $-0.09 \pm 0.20$ \\
\hline $\begin{array}{c}\text { Id2-Bs1 } \\
\text { R436L }\end{array}$ & $+155.75 \pm 0.35$ & $+156.13 \pm 1.04$ & $+155.94 \pm 1.10$ & $+148.38 \pm 0.13$ & $+149.39 \pm 0.17$ & $+148.89 \pm 0.21$ & $+7.05 \pm 1.12$ \\
\hline $\begin{array}{c}I d 2-B s 1 \\
\text { Y458C }\end{array}$ & $+20.88 \pm 0.31$ & $+23.59 \pm 0.46$ & $+22.24 \pm 0.55$ & $+19.15 \pm 0.09$ & $+22.04 \pm 0.90$ & $+20.60 \pm 0.90$ & $+1.64 \pm 1.06$ \\
\hline $\begin{array}{c}I d 2-B s 1 \\
\text { G488R }\end{array}$ & $-160.23 \pm 0.40$ & $-150.31 \pm 1.44$ & $-155.27 \pm 1.49$ & $-159.62 \pm 0.15$ & $-159.95 \pm 0.11$ & $-159.79 \pm 0.19$ & $+4.52 \pm 1.51$ \\
\hline $\begin{array}{c}\text { Id2-Bs2 } \\
\text { A413T }\end{array}$ & $-29.50 \pm 0.10$ & $-27.54 \pm 0.27$ & $-28.52 \pm 0.29$ & $-29.33 \pm 0.09$ & $-26.31 \pm 0.44$ & $-27.82 \pm 0.45$ & $-0.70 \pm 0.53$ \\
\hline $\begin{array}{c}I d 2-B s 2 \\
\text { V416I }\end{array}$ & $+20.05 \pm 0.07$ & $+20.60 \pm 0.14$ & $+20.33 \pm 0.16$ & $+20.18 \pm 0.05$ & $+21.02 \pm 0.12$ & $+20.60 \pm 0.13$ & $-0.27 \pm 0.20$ \\
\hline $\begin{array}{c}I d 2-B s 2 \\
\text { R436L }\end{array}$ & $+151.51 \pm 0.57$ & $+161.00 \pm 1.97$ & $+156.25 \pm 2.05$ & $+148.38 \pm 0.13$ & $+149.39 \pm 0.17$ & $+148.89 \pm 0.21$ & $+7.36 \pm 2.06$ \\
\hline $\begin{array}{c}I d 2-B s 2 \\
Y 458 C\end{array}$ & $+21.33 \pm 0.81$ & $+22.71 \pm 0.28$ & $+22.02 \pm 0.86$ & $+19.15 \pm 0.09$ & $+22.04 \pm 0.90$ & $+20.60 \pm 0.90$ & $+1.42 \pm 1.25$ \\
\hline $\begin{array}{c}I d 2-B s 2 \\
\text { G488R }\end{array}$ & $-159.95 \pm 0.24$ & $-155.89 \pm 0.72$ & $-157.92 \pm 0.76$ & $-159.62 \pm 0.15$ & $-159.95 \pm 0.11$ & $-159.79 \pm 0.19$ & $+1.87 \pm 0.78$ \\
\hline
\end{tabular}

